\title{
Die DGIM als Garant der Fort- und Weiterbildung in der gesamten Inneren Medizin
}

Die Deutsche Gesellschaft für Innere Medizin (DGIM), 1882 in Wiesbaden gegründet und damit eine der ältesten medizinischen Fachgesellschaften, möchte die wissenschaftlich fundierte und evidenzbasierte Fort- und Weiterbildung in der gesamten Breite der Inneren Medizin fördern. Die DMW - seit 1995 Organ unserer Gesellschaft - ist eine der ältesten und renommiertesten medizinischen Fachzeitschriften im deutschen Sprachraum und widmet sich demselben Ziel. Vergleichsweise jung sind dagegen die 2011 eingeführten DGIM-Gesellschaftsausgaben der DMW: In jedem Heft sind alle elf Schwerpunkte der DGIM mit einem aktuellen Fachbeitrag vertreten. Die DGIM und die von ihr vertretene gesamte Innere Medizin sieht sich als Summe ihrer 11 Schwerpunkte und deren Gesellschaften, möchte aber auch mehr sein, einen sogenannten „added value“ liefern. Die wissenschaftlichen Jahrestagungen der Schwerpunktgesellschaften präsentieren zunehmend die originalwissenschaftlichen Daten und vermitteln wissenschaftliche Fortbildung auf höchstem Niveau; ähnlich verhält es sich mit den wissenschaftlichen Zeitschriften der Schwerpunktgesellschaften. Wo ist hier die Genuität der DGIM zu suchen und somit die Legitimation für die Herausgabe solcher DGIM-Gesellschaftsausgaben?

Die DRG-getriebene Ökonomisierung und Kommerzialisierung der Medizin hat u.a. auch dazu geführt, dass vor allem die Durchführung von Prozeduren und Interventionen finanziert wird, seien sie diagnostischer oder therapeutischer Natur. Intellektuelle Leistungen, auch wenn sie noch so bedeutsam und kostensparend für den individuellen Patienten oder das Gesundheitssystem sind, werden nicht honoriert. Ich denke hierbei an die Differenzialdiagnose „Fieber unklarer Genese“ oder „Gewichtsverlust unklarer Genese“ oder „Entzündliches Krankheitsbild unklarer Ätiologie“ oder „Chronic Fatigue Syndrom“. Der Umgang mit solchen Patienten erfordert vor allem auch fundierte Kenntnisse in der gesamten Inneren Medizin. Und gerade hier liegt ein essenzielles Ausbildungspotenzial für Assistenten, ein Ausbildungsauftrag, der durch die Ökonomisierung und das schlanke „case management“ nicht unerheblich be- und verhindert werden kann. Die Kommission für Krankenversorgung der DGIM hat in ihrem vielbeachteten Bericht herausgearbeitet, dass die Innere Medizin das im stationären Bereich am weitesten verbreitete Fachgebiet ist. Dies ist sicher keine Überraschung; fast an allen Krankenhäusern gibt es Fachabteilungen für Innere Medizin. Für mich überraschend aber war das Ergebnis, dass 60\% aller internistischen stationären Fachabteilungen den Namen „Allgemeine Innere Medizin“ tragen. Dies bedeutet, dass wir sowohl eine wissenschaftliche Gesellschaft für Innere Medizin (DGIM) als auch Fort- und Weiterbildung in der gesamten Inneren Medizin brauchen. Zudem brauchen wir auch einen Facharzt für (Allgemeine) Innere Medizin, der schon einmal von einem Deutschen Ärztetag abgeschafft wurde - gegen den Willen der betroffenen wissenschaftlichen Fachgesellschaft, der DGIM, und des zuständigen Berufsverbandes, des BDI. Auch alle Schwerpunktfachärzte werden weiterhin eine Fort- und Weiterbildung in allgemeiner Innerer Medizin benötigen.

In der ambulanten Medizin wird der Mangel an Hausärzten vor allem auf dem Land angeprangert. Die „Allgemeinärzte der stationären Medizin“ sind die allgemeinen Internisten bzw. die Internisten mit einem breiten Wissen in der Inneren Medizin. Aufgabe der DGIM muss es sein, die Breite der Inneren Medizin nicht nur wissenschaftlich zu repräsentieren und berufspolitisch mit dem BDI in Partnerschaft zu vertreten, sondern auch die Breite der klinischen Fort- und Weiterbildung zu erhalten und darzustellen. Die DGIM-Jahrestagungen in Wiesbaden sind hier ein gutes Beispiel: Sie haben sich als der industrieunabhängige Weiterbildungskongress für das gesamte Gebiet der Inneren Medizin etabliert. Hier können Ärzte in Weiterbildung zum Internisten oder zum Schwerpunktfacharzt notwendiges Wissen für die Facharztprüfung erwerben, aber auch Fachärzte, Oberärzte und Chefärzte können sich auf allen Gebieten der Inneren Medizin auf den aktuellen Stand bringen lassen. Vorgetragen und zur Diskussion gestellt werden die Themen von herausragenden Repräsentanten der Schwerpunktgesellschaften.

Dieses Angebot sollten die DGIM-Mitglieder mehr als bisher in Anspruch nehmen, zumal die Kongressteilnahme in Wiesbaden im Jahresmitgliedsbeitrag enthalten ist. Obwohl die Jahrestagungen der DGIM von inzwischen mehr als 8500 Teilnehmern besucht werden, sind darunter nur etwas mehr als $15 \%$ der über 22000 DGIM-Mitglieder vertreten. Dabei ergänzen sich die Jahrestagungen der DGIM und ihrer Schwerpunkte komplementär. Gleiches gilt für die Fort- und Weiterbildung der DGIM in ihren Publikationsorganen, den sog. Printmedien. Die DGIM-Gesellschaftsausgaben der DMW sollen hierzu einen wesentlichen Beitrag leisten. So sollen aus allen Schwerpunkten der Inneren Medizin 6-mal im Jahr - allgemein verständlich, aber wissenschaftlich fundiert - besonders aktuelle Entwicklungen aus allen Schwerpunkten der Inneren Medizin herausgegriffen und übersichtlich dargestellt werden, dies auch und nicht zuletzt für alle Internisten, die den Anspruch erheben, allgemeine Innere Medizin kompetent vertreten zu können.

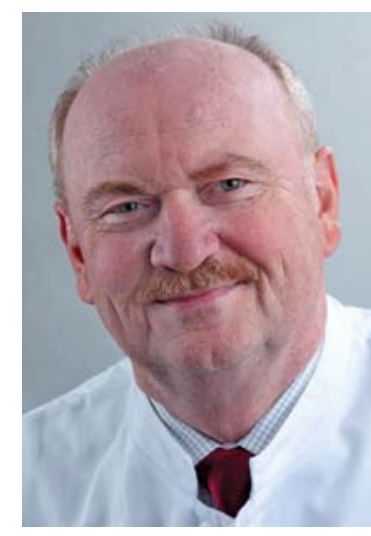

M. P. Manns

Institut

Klinik für Gastroenterologie, Hepatologie und Endokrinologie der Medizinischen Hochschule Hannover

Bibliografie

Dol 10.1055/s-0031-1298881 Dtsch Med Wochenschr 2012; 137: 165 - (c) Georg Thieme Verlag KG · Stuttgart - New York · ISSN 0012-0472

Korrespondenz Univ.-Prof. Dr.

\section{Michael P. Manns}

Direktor der Klinik für Gastroenterologie, Hepatologie und Endokrinologie, Medizinische Hochschule Hannover Carl-Neuberg-Str. 1 30625 Hannover Tel. 0511/532-3306 Fax 0511/532-4896 eMail manns.michael@ mh-hannover.de 